\title{
Simultaneous membranous nephropathy and diabetic nephropathy occurrence in a patient: A case report
}

Received 30 Octomber 2020

Accepted 01 March 2021

Binyao Tian, Nan Liu, Tianhua Xu, Xiaodan Liu, Li Yao*

The Nephrology Depatment of the first hospital of China Medical University, Shenyang, Liaoning Province, China

\section{Abstract}

Membranous nephropathy (MN) is the most common glomerular disease in adults and is constantly associated with the occurrence of nephrotic syndrome. While diabetic kidney disease (DKD) and diabetic nephropathy (DN), which often occur in diabetic patients, are considered as the major causes of end-stage kidney disease. Actually, MN often occurs in patients with diabetes mellitus (DM), but to obtain a clear differential diagnosis without a renal biopsy has become difficult. Here we report the case of a female diabetic patient who developed both MN and DN simultaneously.

Keywords

diabetic nephropathy $\cdot$ membranous nephropathy $\bullet$ tacrolimus

\section{Introduction}

Membranous nephropathy (MN) is the most common glomerular disease in adults and is always associated with the occurrence of nephrotic syndrome [1]. MN is characterized by high levels of proteinuria, edema, hypoalbuminemia, and elevated serum lipids [2]. Typical renal biopsy of MN involves thickening of glomerular basement membrane (GBM) on light microscopy, granular deposits of immunoglobulins (usually IgG4), which is normally expressed on the podocyte membrane antigen PLA2R [3]) and complement (C3) along the capillary walls on immunofluorescence, and subepithelial deposits on electron microscopy examination [1]. MN is a chronic disease, with spontaneous remissions (occurs in about $30 \%$ of patients, usually within the first 2 years after diagnosis in those patients with milder presentations) and frequent relapses. Those who do not undergo remission progress to end-stage renal disease [1].

Diabetic kidney disease (DKD) and diabetic nephropathy (DN) are considered major causes of end-stage kidney disease worldwide [4]. In China, about $10-40 \%$ of diabetic patients suffer from DKD/DN,[5-7] and $1 / 3$ of these will progress to kidney failure.

Actually, MN might occur in patients with diabetes mellitus (DM) [8, 9]. To obtain a clear differential diagnosis of MN or DN has usually become difficult, and therefore, renal biopsy is regarded as the most effective diagnostic method. We herein presented a case report of a female diabetic patient who developed both MN and DN simultaneously.

\section{Case Report}

A 56-year-old female patient, who had undergone treatment for type 2 diabetes about 11 years ago, had poor blood sugar control. Her blood pressure was in the normal range and has no history of heart disease. She has suffered from proteinuria and edema of both lower extremities for about a year but had no relief after active treatment. The levels of urinary protein on admission were $3.837 \mathrm{~g}$ on average, while had normal renal function with creatinine levels of $44 \mu \mathrm{mol} / \mathrm{L}$. But her blood sugar levels remained poor as usual, with $3.51 \mathrm{mmol} / \mathrm{L}$ at fasting and $21.49 \mathrm{mmol} / \mathrm{L}$ at postprandial, and the levels of glycated hemoglobin were $11.70 \%$. What's more, with high levels of urinary protein, a decrease in serum albumin levels occurred as well. Others, like hemoglobin, blood coagulation indicators, etc, were at normal levels. In terms of physical examination, electromyography revealed diabetic peripheral neuropathy but showed no obvious abnormalities on fundus examination.

After admission, the patient was given symptomatic treatment to control blood sugar levels and reduce proteinuria. After that, a kidney biopsy was performed, which showed diffuse thickening of the GBM, podocyte swelling and vacuolar degeneration, renal tubular epithelial cell granule vacuolar degeneration, and vitreous degeneration of renal arterial wall. MASSON staining shows phenophilin deposits granularly under the epithelium (Figure 1). PAS staining shows mild proliferation of mesangial cells and mesangial matrix, and thickening of the glomerular balloon wall (Figure 2). However, PASM staining showed no obvious spike formation (Figure 3). Immunofluorescence results showed deposition of IgG and PLA2R on the glomerular 


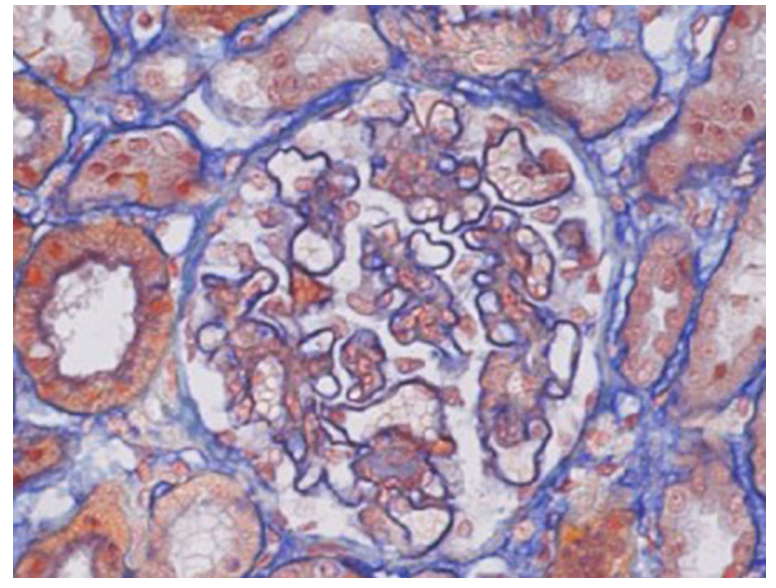

Figure 1. MASSON staining shows granular pheotrophin deposits under the epithelium.

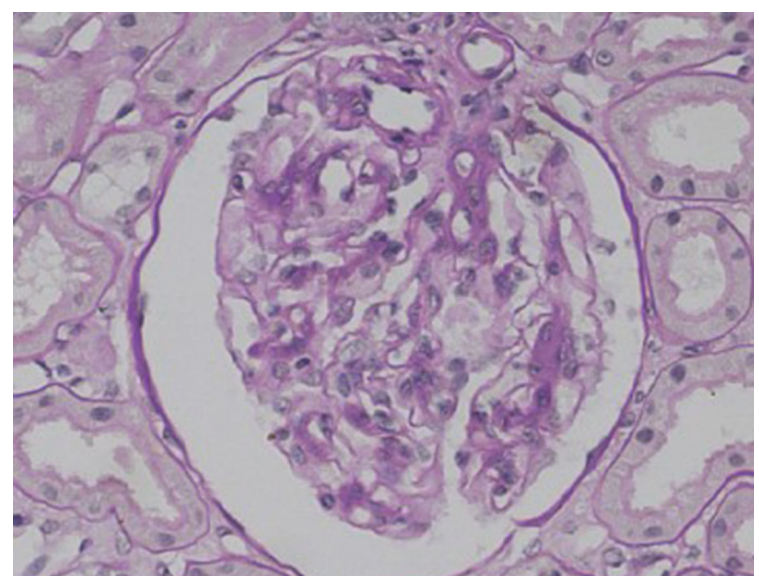

Figure 2. PAS staining shows mild proliferation of mesangial cells and mesangial matrix, and thickening of the glomerular balloon wall.

capillary wall (Figure 4 [left]). Therefore, she was diagnosed with $\mathrm{MN}$ and DN simultaneously.

Based on pathological and clinical manifestations, she was given a treatment of $1 \mathrm{mg}$ of tacrolimus orally twice a day, and active hypoglycemic treatment was given at the same time. After discharge, she was regularly reviewed and followed up. Currently, her urinary protein levels were reduced to about $1 \mathrm{~g}$, blood sugar was under control, and kidney function remained in stable condition.

\section{Discussion}

$\mathrm{MN}$ is the most common indication for renal biopsy in patients with DM.[8, 9] But the symptoms of nephrotic syndrome, such as high levels of proteinuria and edema, suddenly occur in MN patients, while in patients with $\mathrm{DN}$, proteinuria is gradually increased. The urine protein levels in our case were gradually increased, and her proteinuria levels after almost an active treatment for a year still showed no tendency of relief.

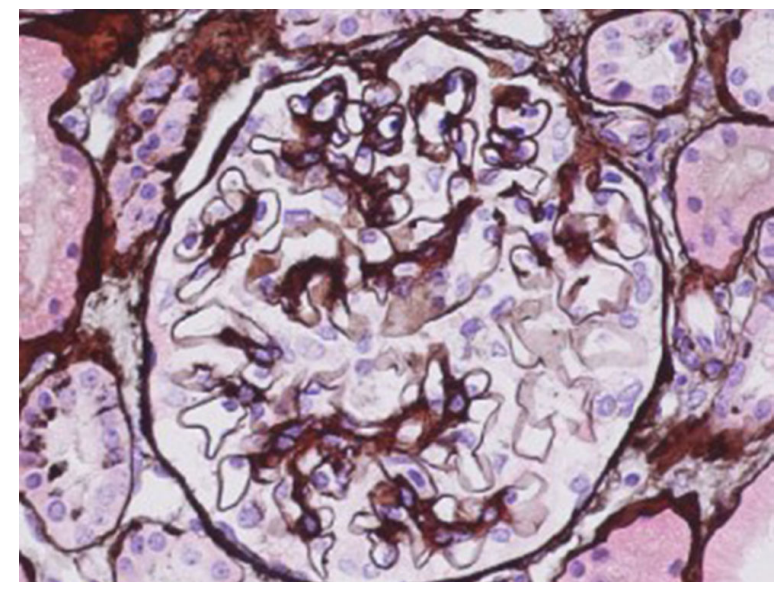

Figure 3. PASM staining shows no obvious spike formation.

Moreover, she had complications of diabetes, such as peripheral neuropathy. These were consistent with the performance of DN. However, according to her renal biopsy, the deposition of IgG and PLA2R on the glomerular capillary wall in immunofluorescence showed the simultaneous occurrence of primary membranous neuropathy (PMN) (Figure 4). As we all know, about $70 \%$ of adult patients with PMN have IgG4 antibodies to podocyte-expressed PLA2R that are present in the circulation and also deposited in glomeruli [10]. And complement components including C3 are also commonly present [11]. Furthermore, established DN is characterized histologically by three lesions, namely mesangial expansion, thickened GBMs, and hyalinosis of afferent and efferent arterioles, [12] which can also be seen on MASSON and PAS staining on this patient (Figures 1 and 2). So, renal biopsy is considered important in diagnosing the condition. Electron microscopy shows that GBM thickening is also a pathological feature of DN, [13] but unfortunately due to technical limitations at the time, this patient did not complete this examination.

The treatment required is more sophisticated. According to the update on DN in 2018, the specific treatment strategies were divided into four major areas: cardiovascular risk reduction, glycemic control, blood pressure control, and inhibition of renin-angiotensin system (RAS) [14]. Olmesartan and insulin were prescribed for DN. For MN, based on the KDIGO Clinical Practice Guidelines, the classical treatment involves cortisol combined with immunosuppression (usually is cyclophosphamide) therapy, [15] and achieves a great effect. However, in patients with DM, cortisol use can disrupt blood sugar control, aggravating the course of MN. Recently, tacrolimus has gradually become one of the main treatment strategies for MN. According to a meta-analysis study [16], tacrolimus was comparable with cyclophosphamide in inducing renal remission in patients with PMN within 1 year. A randomized trial [17] showed that tacrolimus is a very useful therapeutic option for patients with $\mathrm{MN}$ and in preserving renal function. Therefore, in our case, tacrolimus was used to ease her from $\mathrm{MN}$, and her 


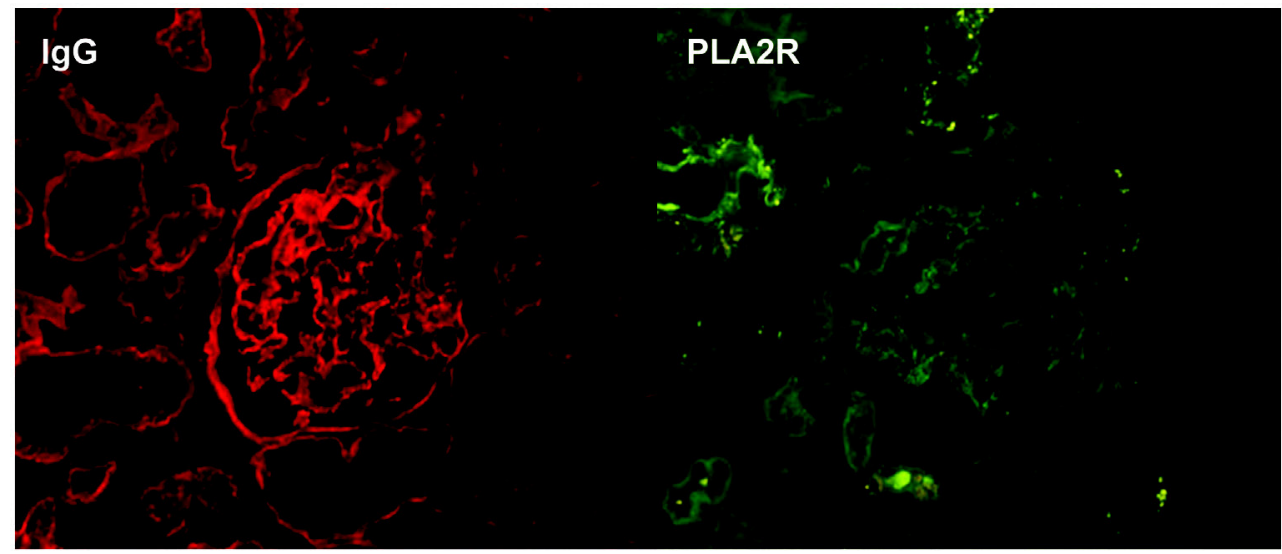

Figure 4. Immunofluorescence microscopy shows deposition of IgG and PLA2R in glomerular capillary wall.

condition has become significantly alleviated.

In conclusion, there are still few patients who suffer from both $\mathrm{MN}$ and DN simultaneously. Their symptoms include a gradual increase of proteinuria and deposition of IgG and PLA2R on the glomerular capillary wall in renal biopsy. Because of
DM, treatment using cortisol remains harmful to control blood sugar levels in the patients. Tacrolimus is regarded as a very useful therapeutic option for patients with $\mathrm{MN}$ and rarely affects blood sugar levels. Therefore, tacrolimus is an effective treatment strategy for patients with both MN and DN. At the same time, RAS can be used to reduce proteinuria.

\section{Funding Sources}

Key R\&D Guidance Plan of Liaoning Province, 2020JH2/10300045 and 2019JH8/10300016; Shenyang City's major scientific and technological innovation research and development plan, 1900192; Liaoning Province Health and Family Planning Appropriate Technology Extension Project, LHATP-201801; National Natural Science Foundation of China, 81770766.

Conflicts of Interest

Li Yao is an Editorial Board Member of the journal. The authors declare no other conflict of interest.

Informed Consent

Patient informed consent has been obtained.

\section{REFERENCES}

[1] Fervenza FC, Sethi S, Specks U. Idiopathic membranous nephropathy: Diagnosis and treatment. Clin J Am Soc Nephrol 2008; 3:905-19.

[2] Lai WL, Yeh TH, Chen PM, Chan CK, Chiang WC, Chen YM. Membranous nephropathy: A review on the pathogenesis, diagnosis, and treatment. J Formos Med Assoc 2015; 114:102-11.

[3] Couser WG. Primary membranous nephropathy. Clin J Am Soc Nephrol 2017; 12:983-97.

[4] Kikkawa R, Koya D, Haneda M. Progression of diabetic nephropathy. Am J Kidney Dis 2003; 41:S19-21.

[5] Zhang L, Wang F, Wang L, Wang W, Liu B, Liu J, et al. Prevalence of chronic kidney disease in China: A cross-sectional survey. Lancet London 2012; 379:815-22.
[6] Lu B, Song X, Dong X, Yang Y, Zhang Z, Wen J, et al. High prevalence of chronic kidney disease in population-based patients diagnosed with type 2 diabetes in downtown Shanghai. J Diabetes Complications 2008; 22:96-03.

[7] Guo K, Zhang L, Zhao F, Lu J, Pan P, Yu H, et al. Prevalence of chronic kidney disease and associated factors in Chinese individuals with type 2 diabetes: Cross-sectional study. J Diabetes Complications 2016; 30:803-10.

[8] Soleymanian T, Hamid G, Arefi M, Najafi I, Ganji MR, Amini M, et al. Non-diabetic renal disease with or without diabetic nephropathy in type 2 diabetes: Clinical predictors and outcome. Ren Fail 2015; 37:572-75. [9] Pham TT, Sim JJ, Kujubu DA, Liu ILA, Kumar VA. Prevalence of nondiabetic renal disease in diabetic patients. Am J Nephrol 2007; 
27:322-28.

[10] Beck Jr. LH, Bonegio RGB, Lambeau G, Beck DM, Powell DW, Cummins TD, et al. M-type phospholipase A2 receptor as target antigen in idiopathic membranous nephropathy. N Engl J Med 2009; 361:11-21.

[11] Ma H, Sandor DG, Beck Jr. LH. The role of complement in membranous nephropathy. Semin Nephrol 2013; 33:531-42.

[12] Najafian B, Alpers CE, Fogo AB. Pathology of human diabetic nephropathy. Contrib Nephrol 2011; 170:36-47.

[13] Drummond K, Mauer M. The early natural history of nephropathy in type 1 diabetes II. Early renal structural changes in type 1 diabetes. Diabetes 2002; 51:1580-7.

[14] Umanath K, Lewis JB. Update on diabetic nephropathy: Core curriculum 2018. Am J Kidney Dis 2018; 71:884-95.

[15] KDIGO. KDIGO clinical practice guideline for glomerulonephritis. Kidney Int 2012; 2:139-74.

[16] Zhu LB, Liu LL, Yao L, Wang LN. Efficacy and safety of tacrolimus versus cyclophosphamide for primary membranous nephropathy: A meta-analysis. Drugs 2017; 77:187-9.

[17] Praga M, Barrio V, Juárez GF, Luño J, Membranosa GEDEDLN. Tacrolimus monotherapy in membranous nephropathy: A randomized controlled trial. Kidney Int 2007; 71:924-30. 